Sir,

\section{Statistical analysis of agreement in measurement comparison studies}

We read the article by Musadiq et $a l^{1}$ with great interest. The authors compare linear dimensions on fundus photographs between digital and photographic film systems. The authors state that due to high correlation coefficients between both measurements, bias was negligible for software correctly set at $35^{\circ}$ field. However, while a high correlation between the two methods makes random operator-error bias unlikely, it cannot account for any systematic (nonrandom) bias occurring.

In comparing agreement between two methods of measurement, one would expect a random scattering of data between the upper and lower 'limits of agreement' $( \pm 2 \mathrm{SD}) \cdot{ }^{2,3}$ However, both Figures 2 and 3 reveal an almost perfect linear increase in the discrepancy between the two measurement methods with increasing distance measured that could be described using a linear regression model. Rather than this representing a mere 'tendency of the measurements to be less accurate with increasing distances measured' as the authors state, this reveals a systematic measurement bias in the authors' study, with digital measurements overestimating the standard photographic measurements by a fixed percentage of the distance measured. This does not reveal agreement between the two methods, but merely association.

In addition, the authors have incorrectly plotted the 'limits of agreement' method as referred to in their text. ${ }^{2}$ This graphical method plots the difference between the two methods against the mean value of the two methods. It is a mistake to plot the difference against either value separately as the authors have, because the difference will be related to each value, a well-known statistical artefact. $^{4}$

This study highlights the inherent dangers of using correlation in assessing agreement between methods of measurement, a fact strongly emphasised by the statistical papers the authors refer to, ${ }^{2,3}$ as well as others. ${ }^{5}$ These methodological errors bring into question the authors' conclusions.

\section{References}

1 Musadiq M, Patsoura E, Hughes S, Yang YC. Measurements of linear dimensions on fundus photographs: comparison between photographic film and digital systems. Eye 2003; 17(5): 619-622.

2 Bland JM, Altman DG. Statistical methods for assessing agreement between two methods of clinical measurement. Lancet 1986; 1(8476): 307-310.

3 Lee J, Koh D, Ong CN. Statistical evaluation of agreement between two methods for measuring a quantitative variable. Comput Biol Med 1989; 19(1): 61-70.
4 Gill JS, Zezulka AV, Beevers DG, Davies P. Relation between initial blood pressure and its fall with treatment. Lancet 1985; 1(8428): 567-569.

5 Ludbrook J. Statistical techniques for comparing measurers and methods of measurement: a critical review. Clin Exp Pharmacol Physiol 2002; 29(7): 527-536.

N Patton, T Aslam

Department of Ophthalmology

Princess Alexandra Eye Pavilion

Chalmers Street

Edinburgh EH3 9HA, UK

Correspondence: N Patton

Tel: + 01315361000

Fax: 01315361574

E-mail: niallpatton@hotmail.com

Eye (2005) 19, 363. doi:10.1038/sj.eye.6701487

Published online 23 July 2004

Sir,

Microscopic polyangitis presenting with sub-acute reversible optic neuropathy

Microscopic polyangitis (MPA) is a systemic necrotizing nongranulomatous vasculitis that affects small blood vessels. Clinical features are frequently constitutional symptoms like fever, anorexia, weight loss, fatigue, and renal failure. In all, $100 \%$ of patients have haematurea and proteinurea. Rapidly progressive glomerulonephritis may occur. ${ }^{1,2}$

Lung involvement manifests with cough, pleurisy, dyspnea, and haemoptysis. Other features like arthralgia, arthritis, purpura, GIT symptoms, and ocular involvement are rare. We report a case of MPA presenting with an optic neuropathy.

An 80-year-old woman was referred to University Hospital Eye Department, with painless deterioration of vision in the right eye over 4 weeks. She reported fatigue and mild hearing impairment. Headaches and jaw claudication were absent and there were no other symptoms to suggest giant cell arteritis.

She had a history of mild asthma, a long-standing history of mild sinusitis, osteoarthritis, and recent onset of urinary tract infection treated with trimethoprim for 1 week.

At presentation, visual acuity was hand movement in the right eye and 6/6 in the left eye with correction.

Anterior segment examination was normal except for a right relative afferent pupillary defect. Other aspects of her ocular examination were normal, in particular both optic discs were pink and normal in appearance (see Figure 1). 
Her ESR was $75 \mathrm{~mm} / \mathrm{h}, \mathrm{C}$ reactive protein $21 \mathrm{mg} / 1(0$ $5 \mathrm{mg} / \mathrm{l}), \operatorname{IgG} 15.4 \mathrm{~g} / 1$ (8.5-15 g/1), IgA $2.6 \mathrm{~g} / 1$ (0.9-4.5 g/1), urea 5.7 , creatinine 114 . She had proteinurea and haematurea. The $24 \mathrm{~h}$ urine collection for Benz Jones protein was negative. CT scan showed a mild thickening of the right optic nerve suggesting optic neuritis, mucosal thickening of the sphenoid sinus, and the other sinuses were normal.

Temporal artery biopsy showed disruption of the elastic lamina, mild inflammatory cells and thickening of the wall (Figure 2).

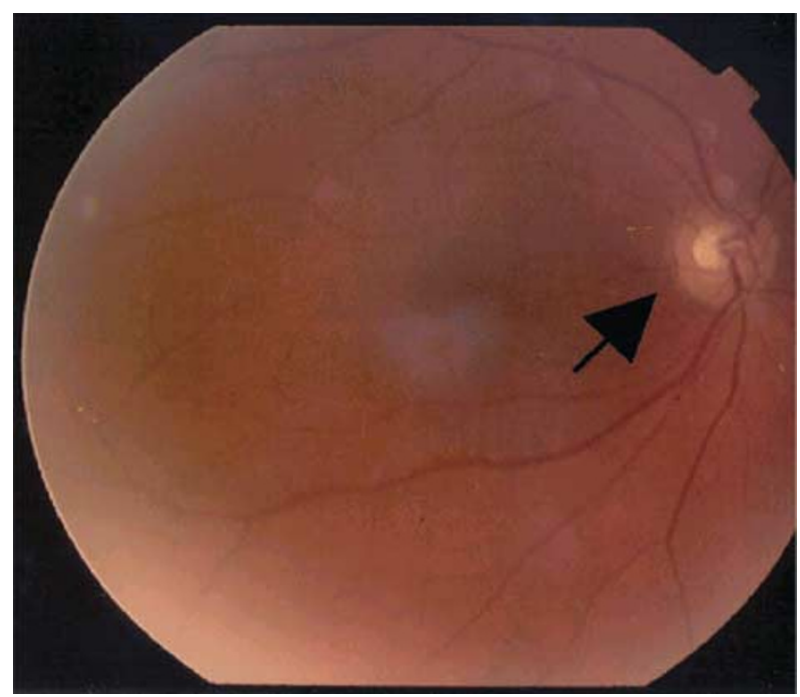

Figure 1 Fundus photograph of the right eye showing the normal appearance of the right optic nerve.

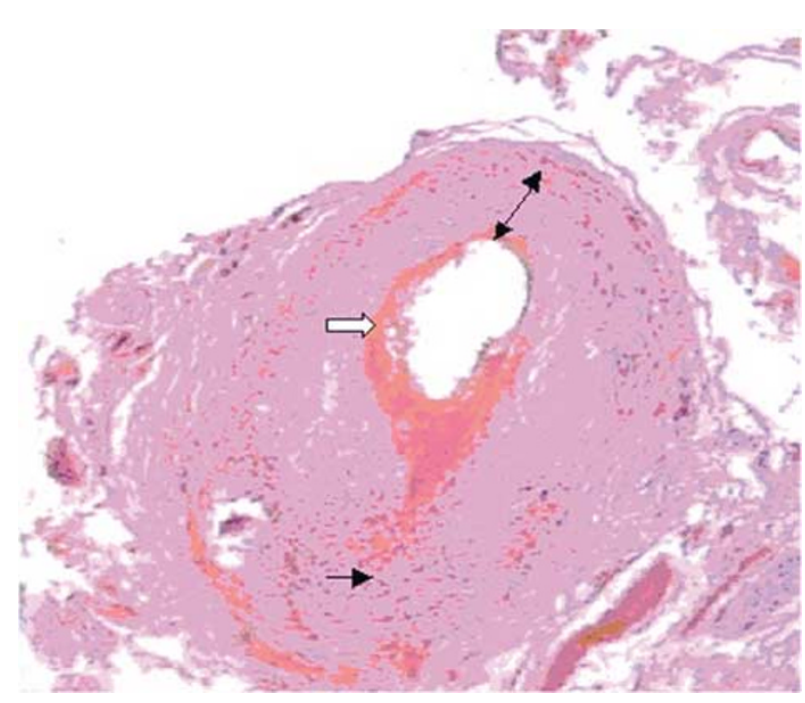

Figure 2 Histological section of the affected segment of the right superficial temporal artery showing thickening and fibrosis of the wall (double arrows) and few inflammatory cells composed of lymphocytes (arrow) and disruption of the elastic lamina (white arrow).
Antineutrophil cytoplasmic antibodies (indirect immunofluorescence for proteinase and myeloperoxidase) were Pr3-ANCA $<5$ (0-10); MYO > 100 (0-10).

Kidney biopsy obtained only a small fragment with sclerosed glomeruli. No evidence of crescent or granuloma was present. The biopsy was inconclusive. She was treated initially with pulsed methylprednisolone $100 \mathrm{mg}$ /day for 4 days, followed by oral prednisolone $60 \mathrm{mg} /$ day and, upon receipt of the results of the investigations that confirmed ANCA positive vasculitis, azothioprine $100 \mathrm{mg}$ was added and the systemic steroids were gradually reduced.

Visual acuity improved to 6/12 after days of treatment. After 4 months, her vision was $6 / 9$ in the right eye, there was no red colour desaturation, and pupil responses were normal. Her hearing also improved significantly.

\section{Comment}

In this case the clinical spectrum of disease and positive MYO-ANCA suggest the diagnosis of MPA.

Antineutrophil cytoplasmic antibodies are positive mainly in small vessel vasculitis. ${ }^{3}$ Therefore, they are positive in Wegners granulamatosis (WG) and MPA and may also be positive in Kawasaki disease, polyarteritis nodosa, systemic lupus (SLE), Crohn's disease, and ulcerative colitis. Two binding specificities are recognized in antinuclear cytoplasmic antibodies: the immunofluorescent cytoplasmic granules with interlobular accentuation (C-ANCA) and the perinuclear pattern (P-ANCA).

The target of C-ANCA is proteinase 3 and of P-ANCA is myeloperoxidase as well as elastase lysozyme.

Clinical association has shown that antiproteinase (C-ANCA) is closely associated with WG (specificity $>90 \%$ ), and antimyeloperoxidase (MPO-ANCA) is associated with microscopic polyangitis (specificity $>90 \%){ }^{1,4}$

The level of ANCA may correlate with disease activity and may be used as a guide in clinical management. ${ }^{2}$ However, ANCA may remain persistently high in some patients despite little clinical evidence of disease activity.

Ocular manifestations of WG are well recognized like scleritis, orbital inflammation, peripheral ulcerative keratitis, keratoconjunctivitis sicca, iritis and less commonly retinal vasculitis and papillitis. ${ }^{5}$

MPA is a relatively newly described condition. From a clinical standpoint, it appears to be very similar to WG; distinguishing it from WG may appear academic, but as the condition becomes better described significant differences may become apparent.

In summary, this 80-year-old lady presented with subacute optic neuropathy due to MPA. Visual loss 
recovered with systemic immunosuppression. The correct diagnosis was important with regard to long-term treatment and prognosis.

Optic neuropathy may be the manifesting sign of small-vessel vasculitis-like MPA. Treatment with a high dose of corticosteroid and immunosuppression may improve the visual outcome.

\section{References}

1 Kaufman AH, Niles JL, Foster CS. ANCA test in ophthalmic inflammatory disease. Int Ophthalmol Clinics 1994; 34(3): 215-227.

2 Ara J. Relationship between ANCA and disease activity in small vessel vasculitis patients with anti MPO ANCA. Nephrol Dial Transplant 1999; 14(7): 1667-1672.

3 Bakkaloglu A, Ozen S, Baskin E, Tinazetepel BN. The significance of antineutrophil cytoplasmic antibody in microscopic polyangitis and classic polyarteritis nodosa. Arch Dis Childhood 2001; 85(5): 427-430.

4 Throne JE, Jabs DA. Ocular manifestation of vasculitis. Rheum Dis Clin North Am 2001; 27(4): 1-12.

5 Pulido JS, Goeken JA, Nerad JA, Sobol WM, Folberg R. Ocular manifestations of patients with circulating antineutrophil cytoplasmic antibodies. Arch Ophthalmol 1990; 108: 845-850.

\section{R Altaie', F Ditizio² and GT Fahy²}

${ }^{1}$ Galway Regional Hospital

Department of Ophthalmology

Galway, Ireland

${ }^{2}$ University College Hospital

Newcastle Road, Galway, Ireland

Correspondence: R Altaie

Tel: 0035351842258

Fax: 0035351842128

E-mail: raltaie2003@yahoo.com

Eye (2005) 19, 363-365. doi:10.1038/sj.eye.6701479

Published online 23 July 2004

Sir,

\section{Photodynamic Therapy of Choroidal Haemangioma Associated with Sturge-Weber Syndrome}

Choroidal haemangioma is an uncommon benign vascular tumour of the choroid that can be either circumscribed or diffuse. ${ }^{1}$ Diffuse choroidal haemangioma is usually part of neuro-oculo-cutaneous haemangiomatosis (Sturge-Weber syndrome). The diffuse choroidal haemangioma of Sturge-Weber syndrome may have localized areas of excessive thickening simulating a circumscribed choroidal haemangioma. ${ }^{2}$

Diffuse choroidal haemangioma can lead to visual loss due to refractive errors, foveal distortion, and exudative retinal detachment. ${ }^{1}$ Tumour regression and resolution of subretinal fluid can be induced with low-dose radiotherapy ${ }^{3}$ or proton beam radiotherapy. ${ }^{4}$ Photodynamic therapy (PDT) is currently being advocated for circumscribed choroidal haemangioma with good short-term results. ${ }^{5}$ In this report we present our initial experience of treating a choroidal haemangioma with PDT in the setting of Sturge-Weber syndrome.

A 31-year-old female who was 15 weeks pregnant and was known to have Sturge-Weber syndrome, presented with reduced vision in the left eye of 6 weeks duration (Figure 1a). On examination the uncorrected visual acuity was $6 / 5$ in the right eye and 6/120 in the left eye. The right eye was normal. Anterior segment examination of the left eye showed prominent episcleral vessels with normal intraocular pressure. Fundus examination revealed diffuse choroidal thickening with a more prominent localized thickening in the temporal quadrant (Figure 1b). The localized component was about $12 \mathrm{~mm}$ in basal dimension and its posterior margin involved the foveola. There was minimal exudative retinal detachment but no cystoid macular oedema. On B-scan ultrasonography the haemangioma had a maximal thickness of $5.6 \mathrm{~mm}$ (Figure 1c).

Following detailed discussions regarding various therapeutic options, it was elected to cautiously observe because of pregnancy. She remained unchanged on clinical and ultrasonographic examination without any spontaneous improvement 4 months following delivery. ${ }^{6}$ Because of concerns for long-term visual loss and encouraged by the response of circumscribed choroidal haemangioma to $\mathrm{PDT}^{7-9}$ she was treated with PDT. A standard dose of verteporfin $\left(6 \mathrm{mg} / \mathrm{m}^{2}\right)$ was given as an intravenous infusion over $5 \mathrm{~min}$ immediately followed by diode laser $(690 \mathrm{~nm})$ applications of $2500 \mu \mathrm{m}$ size, intensity of $600 \mathrm{~mW} / \mathrm{cm}^{2}$, and exposure of $83 \mathrm{~s}\left(50 \mathrm{~mJ} / \mathrm{cm}^{2}\right)$. Altered haemodynamics with high flow through the haemangioma led us to infuse verteporfin over a shorter period ( $5 \mathrm{~min}$ as compared to $10 \mathrm{~min}$ ) and perform PDT soon thereafter instead of waiting for $5 \mathrm{~min}$ after completion of the infusion.

In total, five contiguous spots were applied to the posterior half of the circumscribed portion in a single setting with a Volk PDT lens. On the following day she noted a reduction of vision to hand motions which was recorded on examination a week later. It was attributed to exudative retinal detachment that involved the lower half of the retina and extended up to the foveola. The corrected visual acuity gradually improved over 6 months to $6 / 6$ with complete resolution of subretinal fluid (Figure 1d). Moderate prominence of retinal pigmentation 